\title{
Role of Laser Linewidth in High Speed DWDM System by Incorporating Duobinary Modulation Scheme
}

\author{
Urvashi Bansal \\ Student \\ Department of Electronics and \\ Communication, \\ BBSBEC, Fatehgarh Sahib
}

\author{
Kamaldeep Kaur \\ Assistant Professor \\ Department of Electronics and \\ Communication,
}

\author{
BBSBEC, Fatehgarh Sahib \\ Sushank Chaudhary \\ PhD Scholar \\ Optical Technology Group \\ InterNetworks Research Lab, \\ UUM Malaysia
}

\begin{abstract}
This work is focused on realization of DWDM system of 1.6 $\mathrm{Tb} / \mathrm{s}$ ultra high capacity using duobinary return to zero modulation scheme with EDFA and dispersion compensation scheme. Duo binary format is used as it is suitable for high spectral efficiency at large transmission distance. 40 channels having capacity of 40 Gbps data rate each are multiplexed with channel spacing of $100 \mathrm{GHz}$ to realize $1.6 \mathrm{Tbps}$ as total transmission over optical span of $60 \mathrm{Km}$ by using pre and post compensation technique. The comparison of parameters is observed in terms of $\mathrm{Q}$ value and eye opening keeping BER in acceptable range Moreover, the role of laser line-width is also investigated to minimize the non linearity and four wave mixing effect.
\end{abstract}

\section{Keywords}

DWDM, Laser Line-width, Duobinary Modulation scheme, Pre and Post compensation techniques.

\section{INTRODUCTION}

Due to close channel spacing there is always high possibility of one wavelength effecting the phase and amplitude of another wave. This limits the performance of the optical network. When high power are launched into the fiber these nonlinear effects increase with decrease of channel spacing. Duo binary format gives better performance as this suppresses all the discrete frequency tones that appear in conventional $\mathrm{RZ}$ signal spectrum. According to investigation both NRZ and RZ formats are not suitable for transmission in DWDM system as they are highly susceptible to nonlinear effects. At high data rate of $40 \mathrm{Gbs}$ dispersion compensation fiber having negative dispersion is used to reduce the dispersion in the SMF 16 channels having capacity of $40 \mathrm{Gbps}$ DWDM system is simulated with $25 \mathrm{GHz}$ channel spacing over a transmission distance of $2000 \mathrm{~km}$ using CSRZ, DRZ and MDRZ modulation formats and MDRZ format proved to be most robust against the fiber nonlinearities and optical Kerr's effect[1] .It is demonstrated that pulse-to-pulse interaction and ghost pulses at $40 \mathrm{~Gb} / \mathrm{s}$ can be suppressed by alternating the optical phase of adjacent bits by using either duobinary return-to-zero (DRZ) or a modified-duobinary return-to-zero (MDRZ) modulation format [2]. Both DRZ and MDRZ signals suppress all discrete frequency tones that appear in the conventional RZ signal spectrum. MDRZ has the least timing jitter and amplitude distortion and both of these formats can operate at average channel powers higher than the conventional RZ format [3].It is concluded that the population inversion of EDFA can be controlled by proper choosing of fiber length and injected pump power. The optimum fiber length is 5 meter whereas optimum pump power is $23 \mathrm{~mW}$. The system for 16 channel amplification was designed with 24+-0.3DB intrinsically gain flatness from $1546 \mathrm{~nm}$ to $1558 \mathrm{~nm}$ bandwidth [4]. The output power of $8.4 \mathrm{dbm}$ and average noise figure of $6 \mathrm{db}$ were obtained from this simulation. [5] has presented simulation results of DCF location , optimization for WDM system that use RZ, CRZ and CSRZ modulation schemes. They studied the case of four 10Gbs channel WDM system with uniform DCF placement. With the aid of pre compensation and post compensation, the effect of four wave mixing can also be minimized. The DWDM and pre and post compensation techniques can be employed to increase the efficiency and capacity of optical transmission systems [6]. Non Return to Zero (NRZ) and Return to Zero (RZ) are the most often modulation formats for optical transmission systems. The NRZ format offers narrow optical spectrum as compared to RZ format where as in some cases for long haul communication RZ format is proved as better performance as compared to NRZ formats [7]. However, both RZ and NRZ formats are not suitable for DWDM systems due to presence of non linear effects and FWM. For conventional SMF, the advanced modulation formats such as Duobinary modulation performs well as compared to the NRZ and RZ [3]. In an experiment[8], results shows that CSRZ transmission has best performance because of spectral efficiency and it shows that when partial dispersion compensation is used as in the case in practice to reduce four wave mixing effects the optimization process fail to enhance the performance but with uniform dispersion compensation the results are improved significantly. Till now research has shown that duo binary format is suited for $1.28 \mathrm{~Tb} / \mathrm{s}(32 * 40$ $\mathrm{Gb} / \mathrm{s}$ ) capacity of DWDM system with spacing of $75 \mathrm{GHz}$. Here the work is extended to $1.6 \mathrm{~Tb} / \mathrm{s}$ data $(40 * 40 \mathrm{~Gb} / \mathrm{s})$ for transmission over $60 \mathrm{Km}$ optical fiber by adopting duobinary modulation format and DWDM scheme. For the aid of dispersion compensation, DCF and Erbium doped Fiber amplifier is used in the transmission link. The remainder of paper is stated as: Section II describes the simulation set up, Section III depicts the Result and Section where as conclusion is presented in Section IV.

\section{SYSTEM DESCRIPTION}

To present the results of DWDM system (40 channels at 40 $\mathrm{Gb} / \mathrm{s}$ ) optical systems with multichannel we observe the transmitter waveform at end side of receiver. However, if the bandwidth is reduced too much, there will eventually be intersymbol interference (ISI), as the waveform takes longer to move from one logic level to another. This results in waveform trajectories that begin to close down the eye. The schematic diagram of proposed high speed DWDM based optical transmission system is shown in Fig 1. The proposed model is simulated by using OptiSystemTM software. It enables users to test, plan and simulate almost all type of optical links. In this work, 40 channels with channel spacing of $100 \mathrm{GHz}$ are multiplexed and transported over optical link. The 40 Gbps data is encoded by using Duobinary encoder which is further optical modulated by using Mech Zender 
modulator (MRZ) derived by Continuous wave $(\mathrm{CW})$ laser having power of $00 \mathrm{dBm}$.

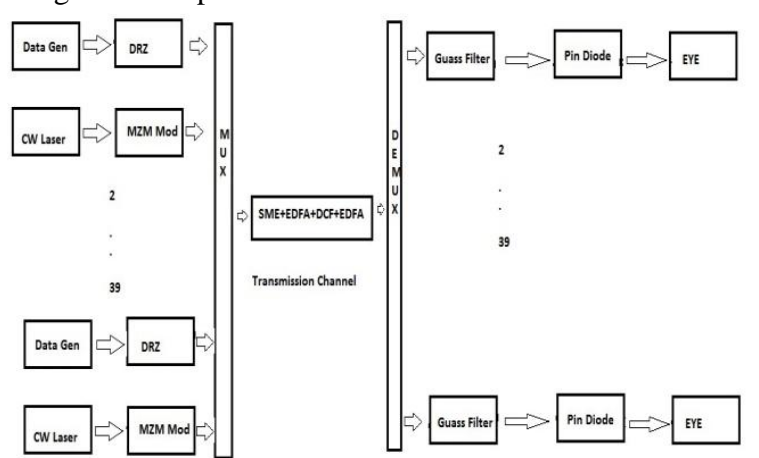

Fig 1: 40 X 40 Gbps Transmission System

The output of MZM is fed to multiplexer which combines the output of all channels to realize the total transmission of 1.6 Tbps data and transported over optical link. It has been shown from Fig 2 (a) \& (b) that after transmission over $50 \mathrm{Km} \mathrm{SMF,}$ the optical power is reduced and non linear effects are more significant.

\section{Optical Spectrum}

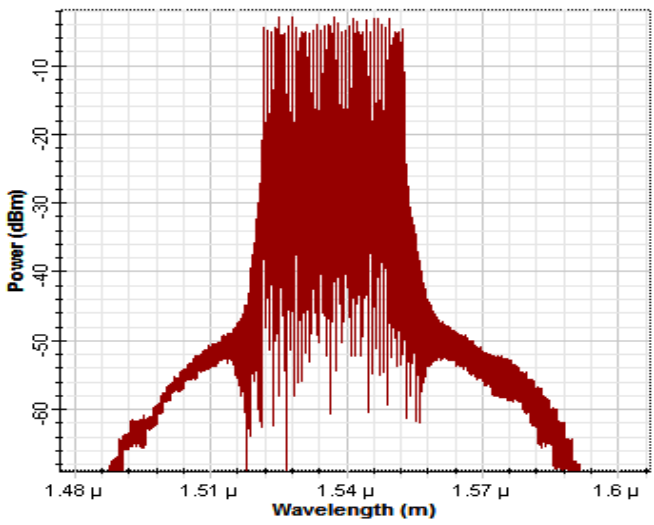

(a)

\section{Optical Spectrum}

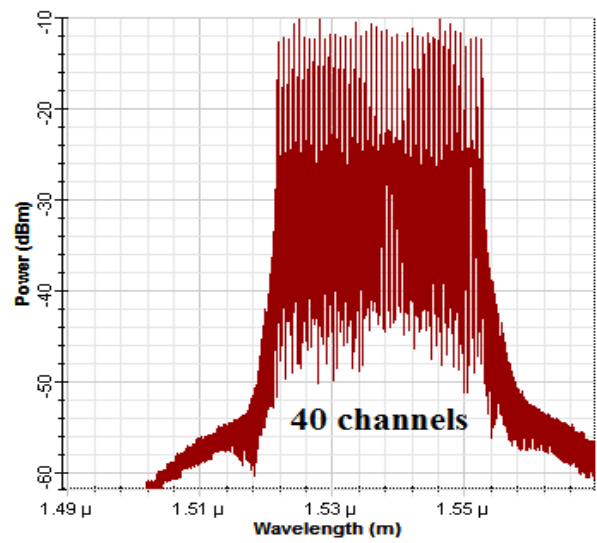

(b)

Fig. 2: Optical spectrum (a) After Multiplexer at transmission side (b) After 50 Km of SMF.

The optical link is comprised of SMF having length of $50 \mathrm{Km}$, EDFA with gain of $10 \mathrm{~dB}$, DCF having length of $10 \mathrm{Km}$ and dispersion of $-85 \mathrm{ps} / \mathrm{nm} / \mathrm{km}$ and again EDFA having gain of
$5 \mathrm{~dB}$. It has been shown from Fig 2 (a) \& (b) that after transmission over $50 \mathrm{Km} \mathrm{SMF}$, the optical power is reduced and non linear effects are more significant. The optical link is comprised of SMF having length of $50 \mathrm{Km}$, EDFA with gain of $10 \mathrm{~dB}$, DCF having length of $10 \mathrm{Km}$ and dispersion of -85 $\mathrm{ps} / \mathrm{nm} / \mathrm{km}$ and again EDFA having gain of $5 \mathrm{~dB}$. In the transmission channel, the positive dispersion of $17 \mathrm{ps} / \mathrm{nm} / \mathrm{km}$ of $50 \mathrm{Km}$ fiber is compensated by using DCF of $10 \mathrm{Km}$ with dispersion of $-85 \mathrm{ps} / \mathrm{nm} / \mathrm{km}$. The output of EDFA is fed to the demultiplexer which splits the optical signal into 40 channels. The optical signal is received by optical Gaussian filter having cut off frequency synchronized with the optical wavelength of each channel at the transmission channel. The output of Gaussian filter is fed to PIN photo diode. The eye diagram is used to measure the output signal at PIN Photo diode.

\section{RESULTS AND DISCUSSIONS}

In this section, the results from the simulation are presented and discussed. The performance of proposed system is evaluated in terms of SNR, total received power and eye diagrams. Fig. 3 depicts the SNR received for channel 1 and channel 40 with respect to line width which also plays significant role in minimizing the non linearity's of optical fiber. The SNR for channel 1 is computed as $64.11 \mathrm{~dB}, 64.43$ $\mathrm{dB}$ and $65.11 \mathrm{~dB}$ at laser line-width of $2 \mathrm{MHz}, 6 \mathrm{MHz}$ and 10 $\mathrm{MHz}$ as shown in Fig 3. (a), which states that there is no significant changes in SNR for the channel 1, when line-width of laser is changed from 2 to $10 \mathrm{MHz}$. whereas for channel 40 , the SNR is computed as $50.11 \mathrm{~dB}, 49.11 \mathrm{~dB}$ and $61 \mathrm{~dB}$ at laser line-width of $2 \mathrm{MHz}, 6 \mathrm{MHz}$ and $10 \mathrm{MHz}$ which states that SNR goes high when the laser line-width changes from 6 $\mathrm{MHz}$ to $10 \mathrm{MHz}$. Similarly, the total power received at photodetector is shown in Fig 3. (b) which clearly states that the received power for channel 1 and channel 40 goes high when the laser line-width changes from $6 \mathrm{MHz}$ to $10 \mathrm{MHz}$. Fig 4 depicts the eye diagram for channel 1, channel 2 , channel 39 and channel 40.

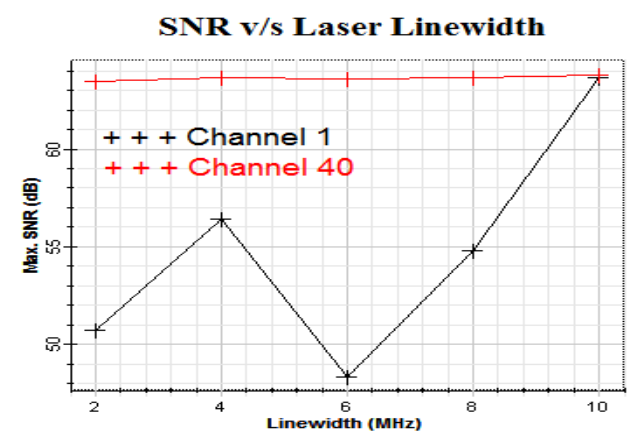

(a)

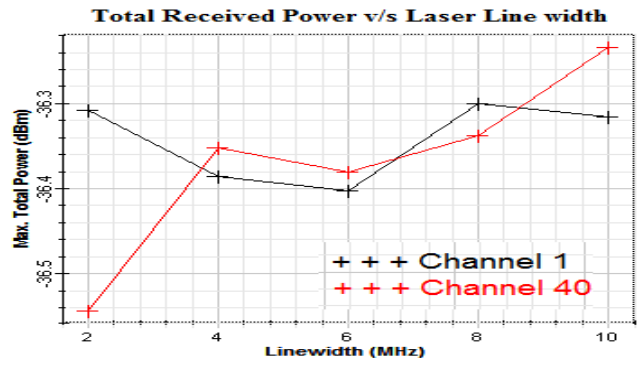

(b)

Fig 3:(a)SNR v/s Laser Linewidth (b)Total received Power v/s Laser linewidth 


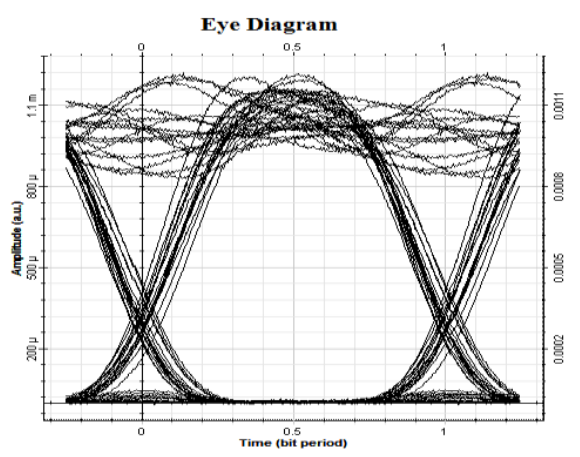

(a)

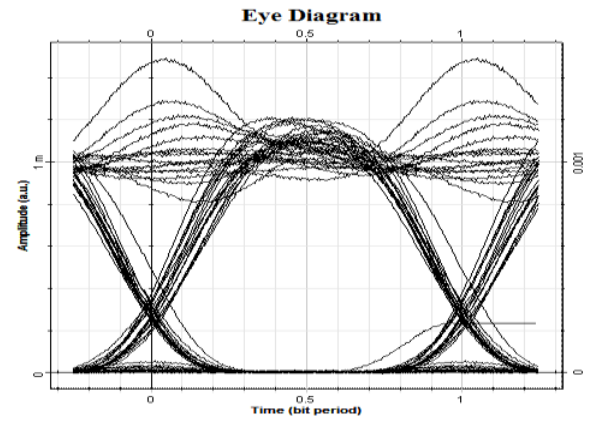

(b)

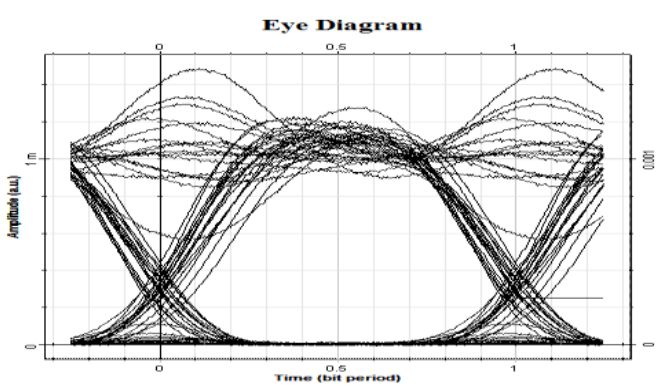

(c)

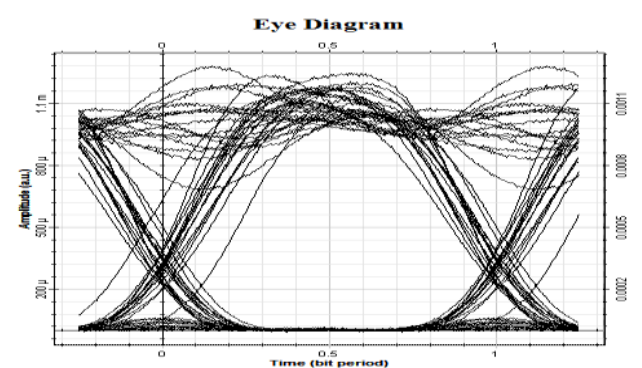

(d)

Fig 4 Eye Diagrams (a) Channel 1 at 2 MHz Laser Linewidth (b) Channel 1 at $10 \mathrm{MHz}$ Laser Line-width (c)

Channel 2 at 2 MHz Laser Line-width (d) Channel 2 at 10 MHz Laser Line-width

The received eye diagrams clearly states that all the channels are successfully transmitted over optical transmission link having span of $60 \mathrm{Km}$.

\section{CONCLUSION}

In this work, Duobinary scheme is proposed as modulation scheme for transmission of 40 channels with $40 \mathrm{Gbps}$ data rate each is over $50 \mathrm{Km} \mathrm{SMF}$ and $10 \mathrm{Km}$ DCF to realize the total transmission of 1.6 Tbps by using DWDM scheme. The channel spacing between all the channels is set to narrow frequency of $100 \mathrm{GHz}$. The role of laser line-width is also investigated as it plays important role to minimize the nonlinearity and four wave mixing. The performance of proposed 40 X 40 high speed system is evaluated in terms of SNR, total received power and eye diagrams which clearly states that all the channels are transmitted up to long optical span of $60 \mathrm{Km}$ with acceptable SNR and BER.

\section{REFERENCES}

[1] Anu Sheetal, Ajay K. Sharma, R.S. Kaler, " Simulation of high capacity $40 \mathrm{~Gb} / \mathrm{s}$ long haul DWDM system using Different modulation formats and dispersion compensation schemes in the presence of Kerr's effect", In: proceedings of the optic 121 (2010)739-749.

[2] K.S. Cheng, J. Conradi, Reduction of pulse-to-pulse interaction using alternative RZ Formats in $40-\mathrm{Gb} / \mathrm{s}$ systems, IEEE Photonics Technol. Lett. 14 (1) (2002) 98.

[3] R.S. Kaler, A.K. Sharma, T.S. Kamal, Simulation results for DWDM systems with ultra-high capacity, Int. J. Fiber Integrated Opt. 21 (5) (2002).

[4] Farah Diana Binti Mahad and Abu Sahmah Bin Mohd Supa,EDFA Gain Optimization for WDM System,Proceedings of Electrika conference,university of Technology,Malaysia,Vol.11,no.1,pp.34-37,2009

[5] Taha Landolsi and Mohamed El-Tarhuni,Optimization of DCF Location for WDM Systems using RZ,Chirped RZ and Carrier-Suppressed RZ Modulation,Innovations in Information Technology,2007.IIT' 07,Proceedings of 4th International November,2007

Conference,pp.646-649,18-20

[6] T. Hoshida, O. Vassilieva, K. Yamada, S. Choudhary, R. Pecqueur, H. Kuwahara, Optimal $40 \mathrm{~Gb} / \mathrm{s}$ modulation formats for spectrally efficient long-haul DWDM systems, IEEE J. Lightwave Technol. 20 (12) (2002) 1989

[7] A. Singh, A.K. Sharma, T.S. Kamal, M. Sharma, Simulative investigations of power penalty for DWDM link in the presence of FWM, Optik-Int. J. Light Electron. Opt, in press, doi:10.1016/j.ijleo. 2008.02.002.

[8] S. Singh, R.S. Kaler, Simulation of DWDM signals using optimum span scheme with cascaded optimized semicon- ductor optical amplifiers, Optik-Int. J. Light Electron. Opt. 118 (2007) 74-82

[9] Ramandeep Kaur and Sushank Chaudhary "Simulative Investigation of Laser Line-width and Channel Spacing for Realization of DWDM Systems under the Impact of Four Wave Mixing" Journal of Optical Communications. Volume 35, Issue 2, Pages 157-165, ISSN (Online) 2191-6322, ISSN (Print) 01734911, March 2014.

[10] G. Bosco, A. Carena, V. Curri, R. Gaudino, P. Poggiolini, On the use of NRZ, RZ, and CSRZ Modulation at $40 \mathrm{~Gb} / \mathrm{s}$ with narrow DWDM channel spacing, J. Lightwave Technol. 20 (9) (2002) 1694.

[11] Shao, Yufeng, et al. "Seamless integration of RZ-DQPSK-DWDM optical links with MISO-OFDM-QPSK system for fourth generation wide-area coverage mobile communication." Microwave and Optical Technology Letters 56.4 (2014): 797-801. 